\title{
A Study on the Effective Gas Safety Management Using Smart Appliances
}

\author{
GyoutaePark $^{1, a}$, Geunjun Lyu ${ }^{2, b}$, Younggyu Kim²,c , Youngdae Kim², \\ and Hiesik $\mathrm{Kim}^{1, \mathrm{e}}$ \\ ${ }^{2}$ Dept. of Electrical Computer Engineering, University of Seoul.korea \\ ${ }^{1}$ Korea Gas Safety Corporation \\ agtparkgs@kgs.or.kr, bjlyu@kgs.or.kr, 'youngdae@kgs.or.kr, dyoungdae@kgs.or.kr, \\ edrhskim@kgs.or.kr
}

Keywords: List the keywords covered Gas safety, sensor, leak, pressure, earthquake.

\begin{abstract}
In this paper, we developed and tested the effective gas safety management system by using wireless intelligent gas safety appliances to monitor gas flow and pressure, earthquake in a micom-gas-meter, to check combustible gas leaks and temperature (upper $100^{\circ} \mathrm{C}$ ) in an automatic extinguisher and to measure smoke and $\mathrm{CO}$ gas. Then our system takes safety measures such as cutoff, warning and messages to protect a serious risk if results of event are abnormal, communicates with a wall-pad including a gateway with ZigBee network in buildings and to inform the event to user via the safety management program in our server. Also, the inner cutoff valve of intelligent gas meters and automatic extinguishers are operated automatically if irregular events are occurred.
\end{abstract}

\section{Introduction}

The new and excellent class apartments are equipped with safety devices in parts of gas, electricity and water facilities. It is clear that sensors will be made of safer and more comfortable human lives in the future. We can use them as not only fire alarms or gas leak detectors, but also biochemical attack watchers [1]. We propose a gas safety management system by using intelligent multi-function gas meters, which include ZigBee network technology, automatic gas leak cutoff, sensors of smoke, methane and temperature. At first we developed the intelligent micom-gas-meter which has a built-in cutoff valve and sensors of flow, pressure and earthquake $[2,8]$. That gas meter operates its inner valve and shut off the gas, serving a warning on users if any abnormal event is occurred for the gas flow, pressure and earthquake. Also, external gas valve is cut off if monitoring temperature of upper $100{ }^{\circ} \mathrm{C}$, smoke for fire and leakage (methane and $\mathrm{CO}$ ) in gas pipeline and facilities [3]. In this paper, we equip wireless modules to gas safety appliances. Those are EM250 ZigBee chips with ZigBee PRO stack [4-7]. Our proposed gas safety management system is configured by the topology of star types with a micom-gas-meter, an automatic extinguisher with a methane detector and a temperature sensor, and two sensors of smoke and $\mathrm{CO}$ [8]. And then controls all the gas safety devices through a wall-pad including a gateway with ZigBee network. If abnormal state is occurred at every sensor, the system takes safety measures to protect a serious risk [9]. And the system inform risk situation of user. So we improved the performance of gas safety Gas safety management system[9], compared with CC2430 chips [3] by adding various functions of an automatic fire extinguisher, sensors of smoke and $\mathrm{CO}$, and remote control via web sites. 


\section{The intelligent Gas Safety Appliances}

A. The intelligent Micom-gas-meter

The intelligent micom-gas-meter, a kind of a general gas meter, built in a micro-controller and a cutoff valve, is not only to measure gas flow and pressure but also to monitor earthquake. This instrument can open and close an inner cutoff valve and serve a warning on users if it is uncommon for flow, pressure and earthquake sensors in gas facilities and houses [2]. Figure 1 shows the appearance of an intelligent micom-gas-meter with a wireless ZigBee communication module including EM250.
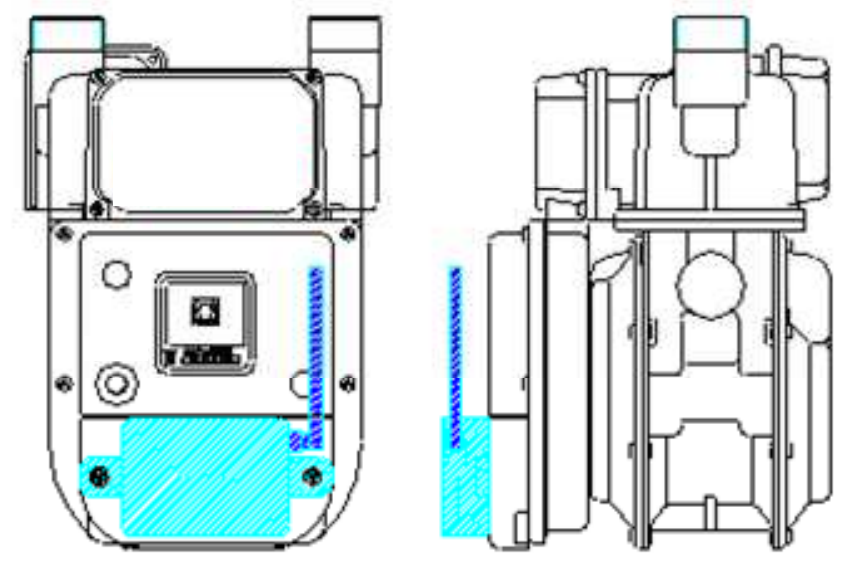

Fig. 1. A micom-gas-meter with wireless modules

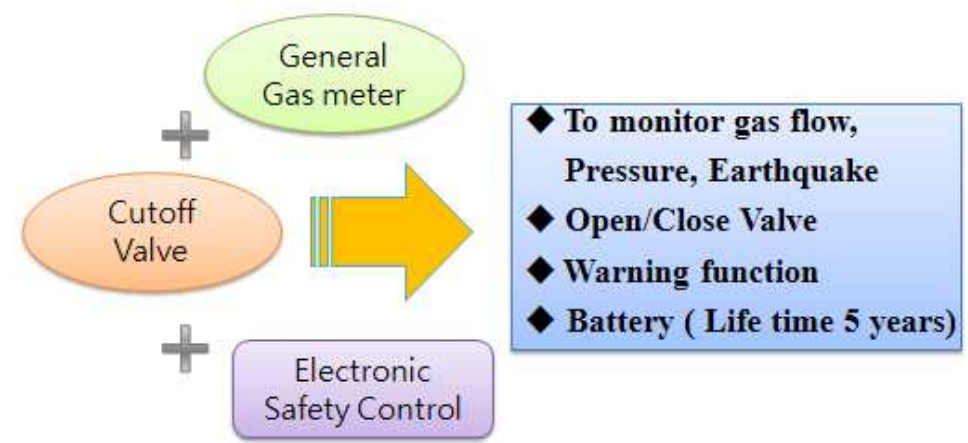

Fig. 2. Components and functions of an intelligent micom-gas-meter

In Figure 2, the components and functions of an intelligent in micom-gas-meter are showed. The micom-gas-meter consists of a general mechanic type gas meter pulus a built-in cutoff valve and electronic safety functions, which measure flow rate of gas, pressure, and earthquake. Flow types are massive, cumulative, instantaneous rising and descending items. Pressure types are mainly high and low pressure components. An earthquake measuring 3.0 on the Richter Scale and alarming, and buzzer functions [2]. Table 1 appears safety functions which a micom-gas-meter can intelligently control itself.

Table 1 Functions of an intelligent micom-gas-meter

\begin{tabular}{|c|l|c|l|}
\hline No. & \multicolumn{1}{|c|}{ Functions } & No. & \multicolumn{1}{|c|}{ Functions } \\
\hline 1 & The mass flow rate $\left(\mathrm{m}^{3} / \mathrm{h}\right)$ & 7 & Set of Upper pressure $\left(\mathrm{mmH}_{2} \mathrm{O}\right)$ \\
\hline 2 & The cumulative flow rate $\left(\mathrm{m}^{3} / \mathrm{h}\right)$ & 8 & Set of lower pressure $\left(\mathrm{mmH}_{2} \mathrm{O}\right)$ \\
\hline 3 & The instantaneous rising flow rate $\left(\mathrm{m}^{3} / \mathrm{h}\right)$ & 9 & The continuous use time $(\mathrm{min})$ \\
\hline 4 & The instantaneous descending flow rate $\left(\mathrm{m}^{3} / \mathrm{h}\right)$ & 10 & Open/Close cutoff valve \\
\hline 5 & The detail flow rate $\left(\mathrm{m}^{3} / \mathrm{h}\right)$ & 11 & Check cutoff valve \\
\hline 6 & The current pressure $\left(\mathrm{mmH}_{2} \mathrm{O}\right)$ & 12 & $\begin{array}{l}\text { Management codes, } \\
\text { Alarming and buzzer }\end{array}$ \\
\hline
\end{tabular}




\section{B. The Automatic Fire Extinguisher}

Figure 3 shows the configuration of an automatic fire extinguisher set. The receiver of an automatic fire extinguisher makes shut off close if any signal is received from a gas leak detector and a temperature sensor indicating 100 degrees. And this receiver makes a fire extinguisher operate and spray fire-extinguishing chemicals if a temperature sensor is received from a signal indicating 130 degrees at the former state. The gas leak detector output $12 \mathrm{~V}$ when to detect combustible gas.

C. The smoke and CO sensors

Smoke and carbon monoxide $(\mathrm{CO})$ sensors can be connected with an automatic fire extinguisher or with a wall-pad directly as Figure 3 .

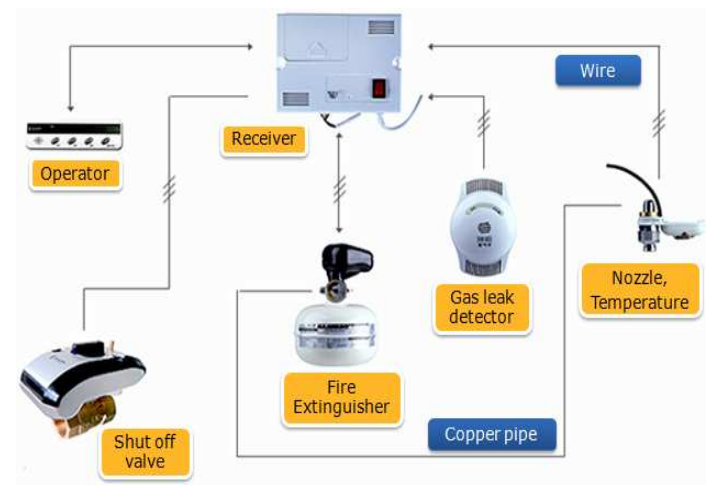

Fig. 1. The configuration of an automatic fire extinguisher

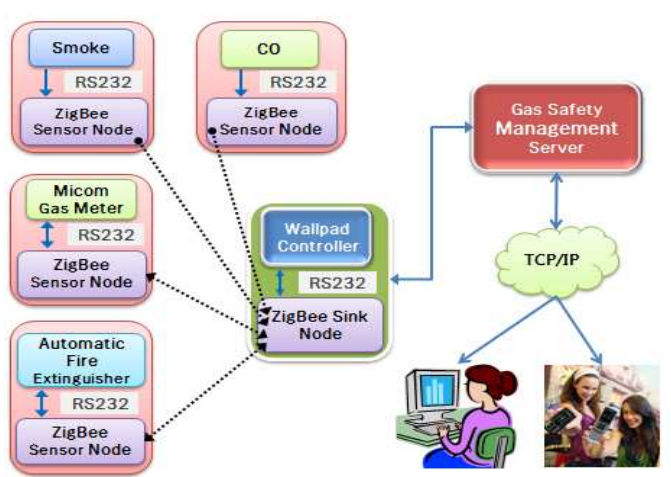

Fig. 2. The network configuration of the gas safety management system extinguisher

D. The wireless modularization of gas safety appliances and Networking

Figure 4 shows the wireless modularization of gas safety appliances such as the micom-gas-meter, an automatic extinguisher and sensors of smoke and CO. Also, Figure 4 appears the diagram of network configuration to operate gas safety management system included in the former gas safety appliances with ZigBee sensor nodes, in the center of a wall-pad controller including a ZigBee sink node connected with a gas safety management server in user's house. That server is connected with a broad band network and users can control their gas safety system. In figure from 4 to 6, Line is the connection of wired communication and a dotted line is that of wireless ZigBee network. We configured ZigBee network as the star topology in one house [6, 7] and controlled all the devices through a wall-pad with gateway function. The gas safety management server communicates with a wall-pad controller trough LAN cable and is connected to the broadband network (TCP/IP) like Figure from 4 to 6. To configure ZigBee network, we used EM250 ZigBee devices and ZigBee PRO stack [6]. A wall-pad is used as an IEC266 (32bits RISC ARM920T, 64MB RAM and NAND Flash).

\section{Gas Safety Management System(GSMS)}

Table 2 shows communication protocols and baud rates in communication for inter-connection. Users able to connect to sever via internet network $(\mathrm{BcN})$ and to verify the operated condition of the function of a micom-gas -meter such as on/off of an inner valve, information of pressure and earthquake sensors, and to control each safety instrument.

Table 2 Baud Rates of Inter-Devices and Nodes

\begin{tabular}{l|l}
\hline \multicolumn{1}{c|}{ Node } & \multicolumn{1}{c}{ Wired-Protocol } \\
\hline \hline Sensor node $\leftrightarrow$ Micom Gas meter & RS-232,4,800 bps \\
Sink node $\leftrightarrow$ Wall-pad & RS-485,9,600bps, Half-duplex \\
Wall pad $\leftrightarrow$ USB port & RS-232,38,400bps \\
Wallpad $\leftrightarrow$ gas safety server & Ethernet $10 \mathrm{Mbps}$ \\
\hline
\end{tabular}


We configured ZigBee communication network in the buildings and those of $\mathrm{BcN}$ to operate gas safety management system in an apartment consisted in tens of micom gas meters, an automatic fire-extinguishing system and sensors of smoke and CO. Signals of gas safety appliances such as micom gas meters, etc., intelligently take steps and are transmitted to users, gas suppliers, and common houses via home gateway if it is abnormal. Users can verify gas safety conditions and control gas safety instruments in their home such as flow rate, pressure, and earthquakes with web pages (Fig. 10). Fig. 5 shows wired and wireless signal flow of the network of a gas safety management system. This system consists of several devices and a server such as sensor nodes (smoke, CO, LNG, temperature, a micom-gas-meter, and an automatic fire-extinguisher), a sink node (a wall-pad), ZigBee and $\mathrm{BcN}$ communication network. In our home, our gas safety management system can monitor gaseous leakage, pressure, flow, and earthquake conditions to protect gaseous exploration and risks. And then that system automatically operate inner safety functions and inform risk state to users if abnormal state. Figure 6 shows a small test bed to experiment operated conditions of three micom-gas-meters, four methane gas detectors, every inner gas valve built in micom-gas-meters and a wall-pad with ZigBee network. We fixed and assembled our gas safety appliances and controllers on the panel. The wall-pad received any information on gas pressure, flow rate and earthquake from micom-gas-meters via wireless ZigBee communication and took a step for the safety.

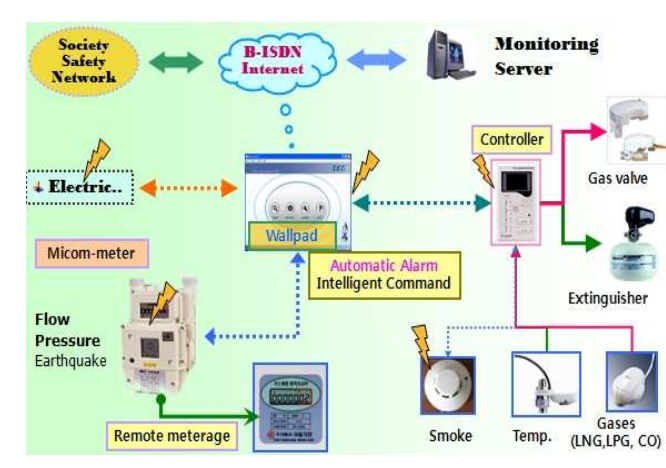

Fig. 5. The signal flow of gas safety management network.

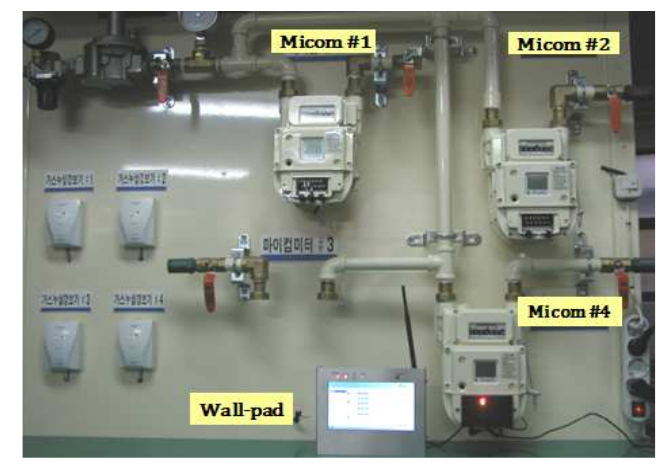

Fig. 6. Experimental devices and Performance Test

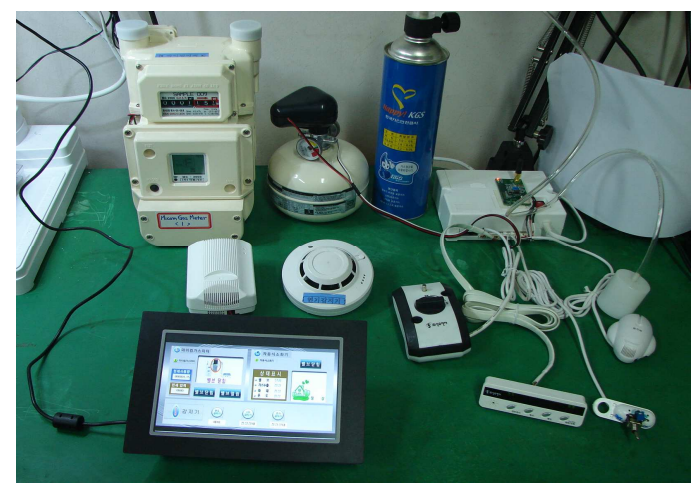

Fig. 7. Control Experiments using a wall-pad

Users and safety managers can verify gas safety conditions and control gas safety instruments in their home through web pages and sites via TCP/IP such as flow rate, pressure, earthquakes, gas leaks, temperature, smoke, and $\mathrm{CO}$ gas at the remote distance. Figure 7 shows several gas safety appliances and a wall-pad with a ZigBee module to control gas appliances and sensors. Figure 8 show the stream diagram to control gas safety appliances by using world wide web(www) pages. Web server specifications are a ML350G6 HP Server with the Xeon Processor E5504, MS Windows7 OS, and MS SQL 2008. In Figure 9, two types of experimental data for flow rates are showed. The state of cumulative flow rate is abnormal state if flow rate exceeds the limit installed in advance. The instantaneous rising flow rate can be occurred if gas pipeline is cracked and flow rate is abruptly 
increased. Table 3 shows procedures and scenarios of gas safety management system based on wireless ZigBee to operate detail functions. Really, our intelligent gas safety managemet have to performe fuctions of when occuring events or unusual states as table 3 . We demonstrated through performance test that our system are normally operated and efficiant for safety management.

Table 3 Functions of the Gas Safety Management System

\begin{tabular}{|c|c|c|}
\hline & Appliances & Functions \\
\hline 1 & $\begin{array}{l}\text { A micom-gas } \\
\text { meter }\end{array}$ & $\begin{array}{l}\text {-Structure : built-in sensors(a pressure, a flow rate, a seism } \\
\text { scope, and an inner gas valve) } \\
\text {-Monitoring : gas pressure and flow rate at pipelines, an } \\
\text { earthquake } \\
\text {-Operating : alarm, shut-off valve }\end{array}$ \\
\hline 2 & $\begin{array}{l}\text { A automatic fire } \\
\text { extinguisher }\end{array}$ & $\begin{array}{l}\text {-Monitoring : combustible gas leak detection, } \\
\text { temperature(100/130 degrees) } \\
\text {-Operating : alarm, shut-off of an external gas valve for gas leak } \\
\text { and above } 100 \text { degrees and close an inner valve in } \\
\text { micom-gas-meter, then spray fire-extinguishing chemicals in } \\
\text { case of above } 130 \text { degrees, and open curtain and windows, } \\
\text { activate a fan }\end{array}$ \\
\hline 3 & A smoke sensor & $\begin{array}{l}\text {-Monitoring : smoke } \\
\text {-Operating : alarm, close an inner valve in a micom-gas-meter } \\
\text { and then stop a boiler system }\end{array}$ \\
\hline 4 & A CO sensor & $\begin{array}{l}\text {-Monitoring : carbon dioxide gas } \\
\text {-Operating : alarm, open curtain and windows, activate a fan, } \\
\text { close an inner valve in a micom-gas-meter, and then stop a } \\
\text { boiler system }\end{array}$ \\
\hline
\end{tabular}

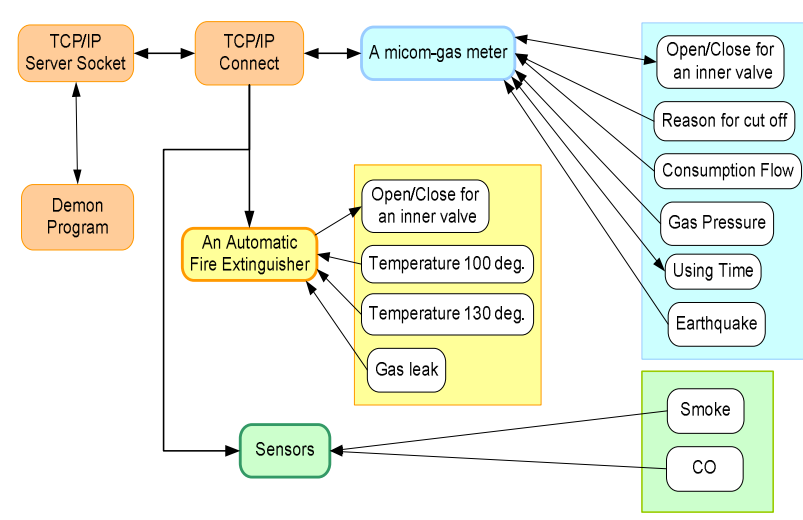

Fig. 8. The remote control of gas safety management system via web sites

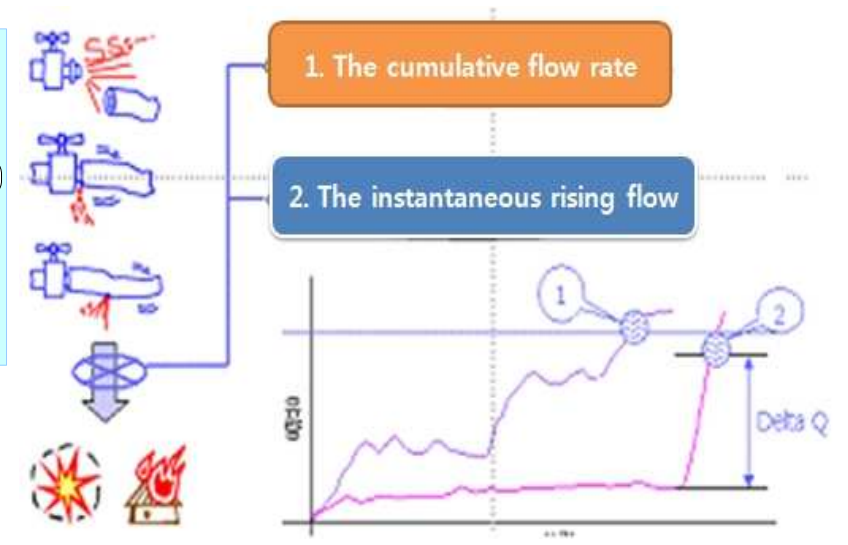

Fig. 9. Experimental results for flow functions.

\section{Conclusion}

We developed and tested the performance of the gas safety management system including gas safety appliances such as a micom-gas-meter, automatic fire-extinguisher and sensors of smoke and CO with built-in ZigBee wireless devices and configured ZigBee network to control and manage risk state on gas facilities in buildings. We verified that gas safety management system by using wireless gas safety instruments can protect an incident effectively and minimize risk ranges. The proposed system takes safety measures while monitoring gas flow rate, gas pressure on pipelines, earthquakes, gas leaks, smoke and temperature for protecting fire, and $\mathrm{CO}$ for monitoring incomplete combustion in parts of gas facilities and then inform dangerous situations to users and managers as a message 
through mobile devices. In near future, components of gas, electric, water, and fire safety instruments can be controlled by a ubiquitous sensor network. Our system, then, can be applied to ubiquitous city infrastructure on gas safety management and rise the efficiency of energy safety and reduction. Hopefully, gas incidents are gradually minimized and then disappeared for our efforts and costs.

\section{Acknowledgment}

This research was supported by the "Research Group of Energy Safety for Next Generation (2010201010095C-21-1-000)" project from MKE (Ministry of Knowledge Economy, Korea) under the program of Energy Technology Innovation

\section{References}

[1] Han Park, "An Enhanced Key Management Scheme Based on Key Infection in Wireless Sensor Network", World Academy of Scicence, Enginerring and Technology 60, pp.249-254, 2009.

[2] http://www.gas-meter.com

[3] G. T. Park, G. J Lyu and Y. G Kim "Implementation of gas safe management system using micom gas-meter with wireless zigbee communication," Korea Information and Communication Society. Vol. 1, pp. 122-123, June 2008.

[4] ZigBee Alliance, ZigBee-2009 Specification : ZigBee Document, 2009

[5] IEEE 802.15.4-2006 standard for wireless personal area networks (WPANs)

[6] Ember Corporation, EmberZNet Application Developer's Reference Manual, 2008

[7] Ata Elahi, Gschwender, "Zigbee Wireless Sensor and Control Network”, Prentice Hall, 2009

[8] Korea Gas Safety Corporation, "2009 The Statistics of Gas incident", 2009.

[9] G.T Park, Y.G Kim and J.R Kwon, "Development of the Gas Safety Management System using an Intelligent Gasmeter with Wireless ZigBee network", World Academy of Science Engineering and Technology(WASET, ISSN: 2070-3724) vol 64, pp. 186-188, Apr, 2010. 